\title{
The 5HTTLPR s allele: a possible mediating variable between environmental factors and affective disorders Xenia Gonda*1,2,3 and Judid Lazary ${ }^{1,3}$
}

Address: ${ }^{1}$ Institute of Pharmacology and Pharmacotherapy, Semmelweis University, Budapest, Hungary, ${ }^{2}$ Clinical Group of Psychiatry, Kutvolgyi Hospital, Semmelweis University, Budapest, Hungary and ${ }^{3}$ National Institute for Psychiatry and Neurology Budapest, Hungary

* Corresponding author

from International Society on Brain and Behaviour: 3rd International Congress on Brain and Behaviour

Thessaloniki, Greece. 28 November - 2 December 2007

Published: 17 April 2008

Annals of General Psychiatry 2008, 7(Suppl I):S60 doi:I0.I I86/I744-859X-7-SI-S60

This abstract is available from: http://www.annals-general-psychiatry.com/content/7/SI/S60

(C) 2008 Gonda and Lazary; licensee BioMed Central Ltd.

Possibilities for research concerning the genetic background of psychiatric disorders and psychological traits has been vastly expanding in the last decade. Amongst several approaches, the 5HTTLPR, a functional polymorphism located in the promoter sequence of the serotonin transporter gene is a likely candidate in delineating genetic factors in the background of affective disorders. The 5HTTLPR polymorphism of the serotonin transporter gene has been found to be associated with both clinical and subclinical forms of depression and anxiety, as well as traits related to neuroticism and affective temperaments carrying a depressive component. Other studies have described its association with biological markers of depression in healthy samples. The s allele has also been described to be associated with resilience and response to stress. Results thus so far indicate that this polymorphism plays a crucial role in the background of affective disorders, while within the boundary of health it is manifested in the form of increased neuroticism or more marked emergence of neuroticism-related traits and in the more pronounced presence of dominant affective temperaments. Although the frequency of the s allele of this polymorphism, which is associated with increased vulnerability towards affective disorders, has been found to be $43 \%$ within the Caucasian population, the lifetime prevalence of unipolar and bipolar depression is around $18-20 \%$. Thus it seems likely that although the presence of this allele predisposes to the development of affective disorders, other factors, such as environmental stress and life events are also necessary for the development of clinical depression. The nature of the manifested affective disor- der is also determined by the interaction of environmental and genetic factors (gene x environment interaction) as well as the interaction of several genes (gene $\mathrm{x}$ gene interaction). Although complex interactions play a governing role in the manifestation of affective symptomatology, the presence of the 5HTTLPR s allele seems to increase vulnerability to environmental influences and stress and thus plays a crucial and permissive role in the development of clinical depression. 